\title{
Prevalence of Infectious Complications in Children with Cancer
}

\section{Zaheer Ullah Khan1*, Nagi Amin², Gaafar Ahmed Mustafa Mohammad1, Sanad Tarmoom1, Mohammad Yousef AlGhar ${ }^{3}$, Anwaar Saud Aldahir ${ }^{3}$, Dayel AlShahrani²}

${ }^{1}$ Department of Paediatric Haematology and Oncology, King Fahad Medical City, Riyadh, Kingdom of Saudi Arabia

${ }^{2}$ Department of Infectious Diseases, King Fahad Medical City, Riyadh, Kingdom of Saudi Arabia

${ }^{3}$ Department of General Paediatric, King Fahad Medical City, Riyadh, Kingdom of Saudi Arabia

Email: ${ }^{\star}$ zaheerullah@kfmc.med.sa

How to cite this paper: Khan, Z.U., Amin, N., Mohammad, G.A.M., Tarmoom, S., AlGhar, M.Y., Aldahir, A.S. and AlShahrani, D. (2019) Prevalence of Infectious Complications in Children with Cancer. Journal of Cancer Therapy, 10, 938-947.

https://doi.org/10.4236/jct.2019.1012080

Received: November 11, 2019

Accepted: December 14, 2019

Published: December 17, 2019

Copyright (๑) 2019 by author(s) and Scientific Research Publishing Inc. This work is licensed under the Creative Commons Attribution International License (CC BY 4.0).

http://creativecommons.org/licenses/by/4.0/

(c) (i) Open Access

\begin{abstract}
Background: Infections are the most common complications during chemotherapy. The trends have been changing over time due to use of multi-agent intensive chemotherapy. Procedure: We looked over our data to see what complications we get in our patients. The data was collected on patients treated in King Fahad Medical City from July 2009 to Dec 2015. Results: We found that 86 patients had one episode only while 92 had 2 or more episodes reaching up to 11 episodes in 1 patient. We found positive cultures in $17.3 \%$ of episodes with staphylococcus as common gram positive and Klebsiella pneumoniae as common gram negative bacteria, respiratory viral infections in $13.8 \%$ and GI infections in $9 \%$. We found Candida albicans as the most common fungus while other yeasts followed. Acute lymphoblastic leukaemia was the most common diagnosis. The episodes were associated with neutropenia of $<500$ in $61.2 \%$ while $58.8 \%$ were associated with duration of neutropenia of $>7$ days in $1^{\text {st }}$ episode and $56.8 \%$ in cases with repeated episodes. We had 2 deaths one from recurrent infections with CNS (Central Nervous System) damage and one from Pulmonary infections causing pulmonary haemorrhage. We had one with persistent neurological sequele from prolonged fungal infection. Conclusions: Neutropenia of $<500$ and duration of $>7$ days came out to be significant risk factors for infection across all diseases. We have an acceptable level of morbidity and mortality due to good supportive services. We need prospective studies to get an up-to-date picture of our practice. We need prophylactic antibiotics and antifungals for high-risk patients like AML.
\end{abstract}

\section{Keywords}

Infections, Cancer, Children 


\section{Introduction}

Infections have caused significant problems in patients with neutropenia over time as the survival improved in cancer due to better and intensified treatments [1]. Life-threatening complications due to bacterial infections have been reported in a significant percentage of febrile episodes in children with cancer [2] [3]. From 1960s to 1980s, aerobic Gram-negative bacilli were the major cause of infection in the neutropenic patient [1]. Schimpff et al. [4] have reported that aerobic Gram-negative bacilli were involved in approximately $60 \%-80 \%$ of the microbiologically proven infections, with $\mathrm{P}$. aeruginosa being a leading isolate and S. aureus was the most important gram-positive isolate. Later, in the mid-80s, the spectrum of organisms causing infection began to change. A steady increase in Gram-positive infections occurred until presently, 60\% - 70\% of bacteremia's with a single organism identified are caused by Gram-positive cocci [5] [6] [7]. Coagulase-negative staphylococci and S. aureus are the predominant organisms. This change from Gram-negative to Gram-positive organisms is due to many factors important among them include aggressive chemotherapeutic regimens causing more severe mucositis, protracted course of neutropenia, almost uniform use of centrally placed catheters, use of Histamine receptor 2 antagonists and use of prophylactic antibacterial agents with relatively weak coverage of Gram-positive organisms [8]. In addition to this the move from Gram-negative to Gram-positive organisms, other Gram-positive organisms have become important causes of infection [5] [9].

Respiratory viruses have become a frequently observed source of infection in about half of the patients with respiratory tract infections in children undergoing chemotherapy [10].

Koskenvuo et al. [11] documented the presence of respiratory infection in $44 \%$ of the cases of children and adolescents with leukaemia and fever, and Srinivasan et al. [12] observed rates of 75\% in their study.

Another important cause of mortality in children with cancer is the invasive fungal infection, especially haematological disorders, who undergo high dose chemotherapy or bone marrow transplant [13] [14] [15]. One of the most common infections is invasive aspergillus's but we also see other non-Aspergillus fungi being increasingly reported [16]. This increase in the incidence of invasive fungal infections in children appears to have increased over the past few decades, due primarily to the prolonged survival of children with immune deficiency syndromes [17] [18] [19].

There are few studies from Middle East and Saudi Arabia showing the same trends but the number is very small and one study looked at only bacterial isolates [20] [21].

Prophylactic antibiotics and antifungals have been shown to reduce the number of episodes of febrile neutropenia and severity of infections especially in cases on intensive chemotherapy and in post-transplant patients [22].

The primary objective of this study was to collect data about our patients to 
see the trends of infections in our set up because we do not have many studies form Middle East and specifically from Saudi Arabia. Also, we wanted to see the effect of the strength of chemotherapy on the trends and to recognize those patients or diseases that will benefit from prophylactic antibiotics and antifungals. This will also help us plan our antibiotic and antifungal policies and reduce the burden on the healthcare system by reducing infection rate and admission rate so reducing hospital days.

\section{Aims and Objectives}

Primary:

To study the prevalence of infections during chemotherapy in our centre as compared to other centres and devise ways to improve it.

Secondary:

To see the effect of degree and duration of Neutropenia on the rate of infection

Inclusion Criteria:

All patients from 0 - 14 years who received chemotherapy at KFMC presenting with fever or positive culture in cases with suspected sepsis.

Exclusion Criteria:

Patients who have comorbidities which predispose to infections e.g. immunodeficiency, down syndrome.

$>$ Non-malignant conditions requiring chemotherapy, i.e. Aplastic anaemia, Congenital and autoimmune Neutropenia, etc.

\section{Materials and Methods}

The data was collected on patients treated (completed treatment) in King Fahad Medical City from July 2009 to Dec 2015. Any patient presenting with a fever of $37.8^{\circ} \mathrm{C}$ on 2 occasions 30 minutes apart or one episode of $38^{\circ} \mathrm{C}$ or a positive culture reported in a child with suspected sepsis presenting with other symptoms i.e. diarrhoea, vomiting, flu, etc. Neutropenia was defined as ANCas < 1500 (mild), $<1000$ Moderate, $<500$ as severe and $<200$ as very severe. Duration of Neutropenia of $<7$ days as mild, 7 - 14 days moderate and $>14$ days as prolonged. The common antibiotics used were Piptazobactam, Amikacin, Vancomycin, Meropenem, Clindamycin, etc. Patients were screened for fungal infections after 5 days of unresolved fever with CT chest, abdomen and pelvis and in some cases with chest $\mathrm{x}$-ray and Ultrasound of the abdomen. The collection of data was from patient files, Hospital Information Management system, CORTEXX (hospital management system) and patient road maps. Specific forms were used for the collection of data by one person and then uploaded on spreadsheets by another so to make sure data is checked by 2 people. Deficient data was rechecked with patient file. The data was analysed, and results compiled which was then compared with other international groups. A comparison was done to see the effect of different chemotherapy regimens on the incidence of infections to formulate a prophylactic antibiotic regimen for those at high risk of infections. 


\section{Statistical Analysis Procedure}

All categorical variables gender, primary diagnosis, site of infections, etc. is presented as numbers and percentages. Continuous variables like age, duration of antimicrobials are expressed as Mean \pm S.D. Pearson's Chi-square/Fisher's exact test is applied according to whether the cell expected frequency is smaller than 5. Independent sample t-test/ANOVA is used to determine the mean duration (days) of antimicrobials in contrast to chemotherapy regimen. In addition, $95 \%$ confidence interval of differences is estimated. P-value $<0.05$ is considered as statistically significant. All data was entered and analysed through statistical package SPSS version 22 .

\section{Results}

A total of around 754 patients were treated from 2009 to 2015. Files of 178 patients were analysed who presented with 480 episodes but the data was not complete in about 75 episodes so 405 episodes were analysed (Table 1).

Age ranged from 3 - 168 months with a mean of $69.5+/-43.3$ months. Male to female ratio is 60:40 respectively (Table 2 ).

$42.5 \%$ of episodes happened at home while $57 \%$ happened in the ward and one each in Emergency room and PICU (Table 3).

86 patients had one episode, 45 had two, 25 had three, while the rest had four or more episodes going up to 11 episodes in one case. Ave episodes per patient were 2.8 in Burkett's lymphoma, followed by 2.6 in ALL, 1.7 in AML, 1.6 in Hodgkin's disease and 1.54 in Medulloblastoma. We had to remove line in $5.2 \%$ (17). ALL comprises $68.5 \%$ of cases followed by AML with 5.5\%, Burkett's lymphoma $4.2 \%$, Medulloblastoma 4.2\%, Hodgkin's disease $4 \%$ and the rest other diseases (Table 4).

Table 1. Summary of the whole study.

\begin{tabular}{cc}
\hline Total Episodes & 483 \\
\hline Incomplete data & 78 \\
Episodes analysed & 405 \\
Total patients screened & 178 \\
Bacterial infections & 72 \\
Viral infections & 56 \\
Fungal infections & 5 \\
PICU admissions & 21 \\
Inotropes & 9 \\
Non-invasive vent & 12 \\
Invasive vent & 13 \\
Mental retardation & 1 \\
Deaths & 2 \\
Central line present & 329 \\
\hline
\end{tabular}


Table 2. Demographic data.

\begin{tabular}{cccc}
\hline Demographics & Male & Female & Total \\
\hline \multirow{2}{*}{ Age } & $70.7+/-42.0$ months & $68.0+/-45.3$ months & $69.5+/-43.3$ months \\
& $(3-168)$ & $(4-161)$ & $(3-168)$ \\
M:F & $101(56.7 \%)$ & $77(43.3 \%)$ & $178(100 \%)$ \\
\hline
\end{tabular}

Table 3. Location of episodes.

\begin{tabular}{cc}
\hline Episodes/Location & No. (\%age) \\
\hline Home & $172(42.5 \%)$ \\
Ward & $231(57 \%)$ \\
PICU & $1(0.2 \%)$ \\
ER & $1(0.2 \%)$ \\
\hline
\end{tabular}

Table 4. Number of episodes per patient with diagnosis.

\begin{tabular}{ccc}
\hline Episodes/Dx & No. of Episodes/Patient (Total) & No./Patient (Average) \\
\hline ALL & $274 / 101$ & $2.6 /$ patient \\
AML & $22 / 13$ & $1.7 /$ patient \\
Burkett's & $17 / 6$ & $2.8 /$ patient \\
Medulloblastoma & $17 / 11$ & $1.54 /$ patient \\
\hline
\end{tabular}

Blood stream was the common infection site with $17.3 \%$ (70), followed by Respiratory tract with $13.8 \%$ (56), Gastroenterology 9.1\% (37), skin and soft tissue $2.5 \%$ (10) and urinary tract, musculoskeletal and CNS 1\% (4) of cases. The yield from blood cultures was $15.8 \%$ and the most common organism was Coagulase negative staphylococcus $3.7 \%$, MRSA $2.2 \%$, Klebsiella $2 \%$, staphylococcus aureus $1.7 \%$, Acinetobacter and enterococcus $1 \%$, followed by other like E. coli, citrobacter, enterbacter seratia and streptococcus species. We also got fungal infections and the leading cause was Candida Albicansin 1.7\%, followed by other yeast $1.2 \%$.

Neutropenia of 1000 - 1500 was found in 28\% of episodes, $500-1000$ in $12.1 \%, 200-500$ in $37.5 \%$ and $<200$ in $22.5 \%$ of episodes (Figure 1). In $59 \%$ of episodes the ANC was $<500$ in $1^{\text {st }}$ episodes while in repeated infections it was $61 \%$ which was significant with a $\mathrm{P}$ value of 0.001 . The duration of neutropenia of $<7$ days was observed in $42.3 \%, 7$ - 14 days in $19.2 \%$ and $>14$ days in $38.4 \%$ of episodes (Figure 2). Blood cultures were positive in $63.4 \%$ cases with duration of Neutropenia exceeding 7 days and $75.6 \%$ in cases with ANC of $<500$. Respiratory infections were more common with duration of $<7$ days in $55.8 \%$ and with ANC of $<500$ at $66 \%$. Skin infections were equally distributed.

21 patients went to PICU needing inotropes in 9 cases and oxygen in 12 cases. 13 cases needed intubation and ventilation. We had two deaths, one from streptococcus pneumonae with concomitant influenza A and B leading to pulmonary 


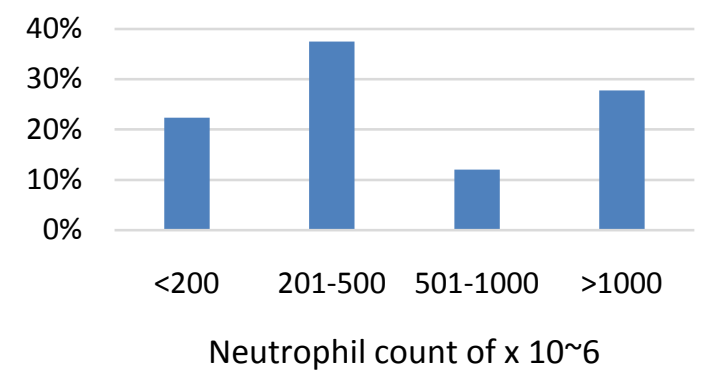

Figure 1. Infection rate with degree of Neutropenia.

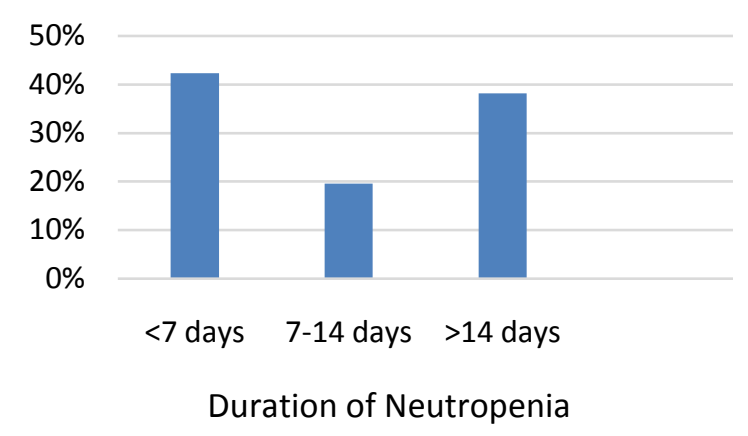

Figure 2. Infection rate with duration of Neutropenia.

haemorrhage. The second case was a case with T cell ALL who had recurrent infections, last one been CNS infection. One patient has long term neurological sequele due to prolonged CNS fungal infection.

The patients were treated according to our febrile neutropenia protocol with Piptazobactam and Amikacin as the first line with addition of Vancomycin if still febrile by day 72 hours and no positive culture followed by Voriconazole/ Amphotericin B as antifungal if still febrile by day 5 without source of infection. Antibiotics were modified according to culture and sensitivity if a culture became positive. Line was removed if recurrent positive culture of same organism or any fungal infection.

\section{Discussion}

The treatment of cancer has been transformed in the last 50 years due to improvement in supportive care services. Infections have always been a major threat to cancer patients because of neutropenia associated with chemotherapy. As time has passed, emergence of resistance in bacteria and fungi has become a major problem in treating high-risk cases that need intensive multiagent chemotherapy and those undergoing transplant. The purpose of this study was to see what our major problems are and how are we doing as compared to others. In 2001, HM Meir et al. [23] from Jeddah presented similar data in acute lymphoblastic leukaemia with Respiratory infection as the common presentation, $24 \%$ had only one episode as compared to $59 \%$ in our study. We found blood stream as the most common site of infection and we had similar results with the Coagulase Negative Staphylococcus as the most common organism. RA Am- 
mann et al. [24] in 2003 from Switzerland had similar findings of 37\% presenting with an infection in 285 episodes as compared to our 45\%. A similar data was presented in 2004 by S Mahmoud et al. [25] from Pakistan with 44\% positive cultures from 62 episodes with blood as the most common site $24 \%$ as compared to our $16 \%$. A recent study from Qatar was published by N AlMulla et al. [26] with the same pattern of organisms as our study with Staphylococcus as the most common gram positive and Klebsiella as the most common gram negative organism. H Ashour and A Alshareef [27] from Cairo reported the same organisms (G negative) in 2009. SS Purewal et al. [28] also reported similar findings from India in 2011. TC Yeh [29] from Taiwan reported in 2014 that prophylactic antibiotics prevented severe infections in high-risk cases thus suggesting a role for prophylactic antimicrobials in these situations.

Sarah Georgaudou et al. [30] from MD Anderson in USA in 2011 reported cases of fungal infections with a difference to our series where they reported aspergillus as the most common while we found Candida and yeasts as the most common organism probably due to different weather conditions or ethnicity. It is apparent from the examples above that we are similar in the way of bacterial infections and the source of infections but different in our fungal organisms.

We have two deaths from infection related complications which is $0.5 \%$ of all the episodes and is excellent as compared to international data as reported by David et al., 2014 from UKALL 2003 trial as 2.4\% and SK Basu et al., from Edinburgh as $3 \%$. It may be partly to the number of less complicated patients as we were building our patient number and we did not have many relapsed patients.

There are some limitations of this study due to the retrospective nature of the study, lack of consecutive case reporting and a lot of missing information, so it may not be representative but none the less presents the overall picture. Therefore, I have decided to extend this study and collect consecutive prospective data so that we can get a clearer picture and make our recommendations on that.

\section{Conclusion}

Infections are still a big problem in cancer patients on chemotherapy and we need to look for other ways to improve our antimicrobial coverage and select cases with high risk and give them prophylactic antibiotics during neutropenic episodes. We need prospective data on our patients to get a clearer picture of the risks facing our patients. We need more education for the patients and their families to bring the child quickly to the hospital and make sure these patients are seen immediately to prevent simple infection progressing to a severe one.

\section{Acknowledgements}

Tariq Wani and Salman for their contribution in helping me analysing the data; Sana Abdullah Research coordinator for preparing the Excel sheet; Shawana Zaheer Research coordinator for data Entry; Bilal Mohammad AlBotoush for data collection are acknowledged. 


\section{Conflicts of Interest}

The authors declare no conflicts of interest regarding the publication of this paper.

\section{References}

[1] Donowitz, G.R., Maki, D.G., Crnich, C.J., et al. (2001) Infections in the Neutropenic Patient-New Views of an Old Problem. Hematology, 1, 113-139. https://doi.org/10.1182/asheducation-2001.1.113

[2] Hann, I., Viscoli, C., Paesmans, M., et al. (1997) A Comparison of Outcome from Febrile Neutropenic Episodes in Children Compared with Adults: Results from Four EORTC Studies. British Journal of Haematology, 99, 580-588. https://doi.org/10.1046/j.1365-2141.1997.4453255.x

[3] Viscoli, C., Moroni, C., Boni, L., et al. (1991) Ceftazidime plus Amikacin versus Ceftazidime plus Vancomycin as Empiric Therapy in Febrile Neutropenic Children with Cancer. Reviews of Infectious Diseases, 13, 397-404. https://doi.org/10.1093/clinids/13.3.397

[4] Schimpff, S.C., Satterlee, W., Young, V.M., et al. (1971) Empiric Therapy with Carbenicillin and Gentamicin for Febrile Patients with Cancer and Granulocyptopenia. The New England Journal of Medicine, 204, 1061-1065. https://doi.org/10.1056/NEJM197105132841904

[5] Zinner, S.H. (1999) Changing Epidemiology of Infections in Patients with Neutropenia and Cancer: Emphasis on Gram-Positive and Resistant Bacteria. Clinical Infectious Diseases, 29, 490-494. https://doi.org/10.1086/598620

[6] Elting, L.S., Rubenstein, E.B., Rolston, K.V.I., et al. (1997) Outcomes of Bacteremia in Patients with Cancer and Neutropenia: Observation from Two Decades of Epidemiological and Clinical Trials. Clinical Infectious Diseases, 25, 247-259. https://doi.org/10.1086/514550

[7] Hughes, W.T., Armstrong, D., Bodey, G.P., et al. (2002) Guidelines for the Use of Antimicrobial Agents in Neutropenic Patients with Cancer. Clinical Infectious Diseases, 34, 730-751. https://doi.org/10.1086/339215

[8] Giamarellou, H. and Antoniadou, A. (2001) Infectious Complications of Febrile Leukopenia. Infectious Disease Clinics of North America, 15, 457-482. https://doi.org/10.1016/S0891-5520(05)70156-2

[9] Cohen, J., Worsley, A.M., Goldman, J.M., et al. (1983) Septicemia Caused by Viridans Streptococci in Neutropenic Patients with Leukemia. The Lancet, 24, 1452-1454. https://doi.org/10.1016/S0140-6736(83)90799-7

[10] Benites, E.C.A., et al. (2014) Acute Respiratory Viral Infections in Pediatric Cancer Patients Undergoing Chemotherapy. Jornal de Pediatria, 90, 370-376. https://doi.org/10.1016/j.jped.2014.01.006

[11] Koskenvuo, M., Mottonen, M., Rahiala, J., Saarinen-Pihkala, U.M., Riikonen, P., Waris, M., et al. (2008) Respiratory Viral Infections in Children with Leukemia. The Pediatric Infectious Disease Journal, 27, 974-980. https://doi.org/10.1097/INF.0b013e31817b0799

[12] Srinivasan, A., Gu, Z., Smith, T., Morgenstern, M., Sunkara, A., Kang, G., et al. (2013) Prospective Detection of Respiratory Pathogens in Symptomatic Children with Cancer. The Pediatric Infectious Disease Journal, 32, e99-e104.

[13] Chamilos, G., Luna, M., Lewis, R.E., et al. (2006) Invasive Fungal Infections in Patients with Hematologic Malignancies in a Tertiary Care Cancer Center: An Autop- 
sy Study over a 15-Year Period (1989-2003). Haematologica, 91, 986-989.

[14] Lehrnbecher, T., Frank, C., Engels, K., Kriener, S., Groll, A.H. and Schwabe, D. (2010) Trends in the Postmortem Epidemiology of Invasive Fungal Infections at a University Hospital. Journal of Infection, 61, 259-265. https://doi.org/10.1016/j.jinf.2010.06.018

[15] Kontoyiannis, D.P., Marr, K.A., Park, B.J., et al. (2010) Prospective Surveillance for Invasive Fungal Infections in Hematopoietic Stem Cell Transplant Recipients, 2001-2006: Overview of the Transplant-Associated Infection Surveillance Network (TRANSNET) Database. Clinical Infectious Diseases, 50, 1091-100. https://doi.org/10.1086/651263

[16] Richardson, M. and Lass-Flörl, C. (2008) Changing Epidemiology of Systemic Fungal Infections. Clinical Microbiology and Infection, 14, 5-24. https://doi.org/10.1111/j.1469-0691.2008.01978.x

[17] Arendrup, M.C., Fisher, B.T. and Zaoutis, T.E. (2009) Invasive Fungal Infections in the Paediatric and Neonatal Population: Diagnostics and Management Issues. Clinical Microbiology and Infection, 15, 613-624. https://doi.org/10.1111/j.1469-0691.2009.02909.x

[18] Steinbach, W.J. (2010) Epidemiology of Invasive Fungal Infections in Neonates and Children. Clinical Microbiology and Infection, 16, 1321-1327. https://doi.org/10.1111/j.1469-0691.2010.03288.x

[19] Tragiannidis, A., Roilides, E., Walsh, T.J. and Groll, A.H. (2011) Invasive Aspergillosis in Children with Acquired Immunodeficiencies. Clinical Infectious Diseases, 54, 258-267. https://doi.org/10.1093/cid/cir786

[20] Meir, H.M., Balawi, I.A., et al. (2001) Fever and Granulocytopenia in Children on ALL Induction Therapy. Saudi Medical Journal, 22, 423-427.

[21] Al-Mulla, N.A., Taj-Aldeen, S.A., et al. (2014) Bacterial Blood Stream Infection and Their Susceptibility Pattern in Children Post Chemotherapy. Infection and Drug Resistance, 7, 289-299. https://doi.org/10.2147/IDR.S70486

[22] Yeh, T.C., Liang, D.C., et al. (2014) Prophylactic Ciprofloxacin and Voriconzole or Micafungin in Children Undergoing Intensive Chemotherapy. Cancer, 120, 1255-1262. https://doi.org/10.1002/cncr.28524

[23] Meir, H.M., Balawi, I.A., Meer, H.M., Nayel, H. and Al-Mobarak, M.F. (2001) Fever and Granulocytopenia in Children with Acute Lymphoblastic Leukemia under Induction Therapy. Saudi Medical Journal, 22, 423-427.

[24] Ammann, R.A., Hirt, A., Luthy, A.R. and Aebi, C. (2003) Identification of Children Presenting with Fever in Chemotherapy-Induced Neutropenia at Low Risk for Severe Bacterial Infection. Medical and Pediatric Oncology, 41, 436-443. https://doi.org/10.1002/mpo.10320

[25] Mahmud, S., Ghafoor, T., Badsha, S.M. and Gul, S. (2004) Bacterial Infections in Paediatric Patients with Chemotherapy Induced Neutropenia. Journal of Pakistan Medical Association, 54, 237.

[26] AAl-Mulla, N., Taj-Aldeen, S.J., El Shafie, S., Janahi, M., AAl-Nasser, A. and Chandra, P. (2014) Bacterial Bloodstream Infections and Antimicrobial Susceptibility Pattern in Pediatric Hematology/Oncology Patients after Anticancer Chemotherapy. Infection and Drug Resistance, 7, 289-299.

[27] Ashour, H.M. and El-Sharif, A. (2009) Species Distribution and Antimicrobial Susceptibility of Gram-Negative Aerobic Bacteria in Hospitalized Cancer Patients. Journal of Translational Medicine, 7, 14. https://doi.org/10.1186/1479-5876-7-14 
[28] Purewal, S.S., Singh, R.P. and Kahlon, R.S. (2011) Study of Bacterial Pathogens and Viral Infections in Neutropenic Cancer Patients. International Journal of Educational Planning \& Administration, 1, 15-22.

[29] Yeh, T.-C., Liu, H.-C., Hou, J.-Y., Chen, K.-H., Huang, T.-H., Chang, C.-Y. and Liang, D.-C. (2014) Severe Infections in Children with Acute Leukemia Undergoing Intensive Chemotherapy Can Successfully Be Prevented by Ciprofloxacin, Voriconazole, or Micafungin Prophylaxis. Cancer, 120, 1255-1262.

[30] Georgaudou, S.P., Pongas, G. Fitzgerald, N.F. et al. (2012) Invasive Mold Infections in Pediatric Cancer Patients Reflect Heterogeneity in Etiology, Presentation, and Outcome: A 10-Year, Single-Institution, Retrospective Study. Journal of the Pediatric Infectious Diseases Society, 1, 125-135. https://doi.org/10.1093/jpids/pis042

\section{Abbreviations}

\begin{tabular}{cc}
\hline CT & Computed Topography \\
CNS & Central Nerves System \\
AML & Acute Myeloid Leukaemia \\
ANC & Absolute Neutrophil Count \\
PICU & Paediatric Intensive Care Unit \\
ALL & Acute Lymphoblastic Leukaemia \\
MRSA & Methicillin Resistant Staphylococcus Aureus \\
\hline
\end{tabular}

